\title{
Über die Vergleichbarkeit von Kookkurrenzverhalten
}

\begin{abstract}
Ein sehr mächtiges Instrument für die Untersuchung von Wörtern und Verwandtschaftsbeziehungen zwischen ihnen ist die Analyse typischer Verwendungskontexte - unabhängig davon, ob die Evidenzen auf Bedeutungskonstitution, ihre Veränderung oder Verwechslung hinweisen, drei Aspekte, die alle bei der Charakterisierung von Parony mie eine Rolle spielen. Auch wenn für die Ermittlung typischer Verwendungsmuster ausgereifte Methoden zur Verfügung stehen, so sollte beim Vergleich der Analysen doch beachtet werden, dass sie diversen Einflussgrößen unterliegen. Neben der Datengrundlage und der Definition und Handhabung des relevanten Kontextes wird im Folgenden besonders darauf eingegangen, welche Rolle verschiedene Teilmengen eines Flexionsparadigmas spielen können, wenn ein Lemma als dessen Gesamtmenge als sprachliche Bezugseinheit einer Untersuchung gewählt wurde. Veranschaulicht wird die Gedankenführung an der beispielhaften Betrachtung von Paronymkandidaten.

The contextual analysis of typical word usage is a powerful instrument for the study of words and their relationships - no matter whether the evidence points to the constitution, change or confusion of meaning, three features which play an important role in characterising paronymy. Although we have sophisticated methods for detecting typical usage patterns, we should be aware that these can be influenced by a number of factors when we try to compare the results of different analyses. Besides the data sample, the definition and treatment of the relevant context, we will look in particular at the role which various subsets of inflectional paradigms can play when a lemma representing the complete paradigm is chosen as the unit of reference for a study. The argumentation will be illustrated with cases of possible paronyms.
\end{abstract}

\section{Einleitung}

Das Institut für Deutsche Sprache bietet ein reichhaltiges Methodenrepertoire, um Wörter korpusbasiert-empirisch zu untersuchen (Teubert/Belica 2014). Mit Hilfe von COSMAS II (Institut für Deutsche Sprache 2017b; Bodmer Mory 2014) kann explorierend im Deutschen Referenzkorpus DeReKo (oder selbst zusammengestellten Teilmengen davon, sog. virtuellen Korpora; Institut für Deutsche Sprache 2017a; Kupietz et al. 2010; Kupietz/ Schmidt 2015) recherchiert werden, Übersichten lassen sich nach verschiedenen Metadaten-Dimensionen auswerten und es können über Kookkurrenzanalysen (Belica 1995) typische Verwendungsweisen aufgespürt werden (siehe Schnörch in diesem Heft). Die Ergebnisse derartiger Analysen, sogenannte Kookkurrenzprofile, betrachten wir als quasi Zusammenfassung der wichtigsten typischen Verwendungsmuster. Bei der Interpretation von Kookkurrenzprofilen lassen sich viele Hinweise auf Elemente entdecken, die zur Konstitution der Bedeutung, zur Differenzierung von Lesarten oder Konnotationen beitragen (Perkuhn 2007a/b; Perkuhn/Keibel 2009; Belica/Perkuhn 2015). Ausgehend von der Idee, dass sich viel über die Beziehung zweier sprachlicher Einheiten über den Vergleich ihrer Kookkurrenzprofile lernen lässt (Belica 2011), bietet sich dieses Mittel der Wahl an, um X-onyme verschiedener Art, insbesondere natürlich auch Synonyme oder Paronyme, zu untersuchen (Storjohann/Schnörch 2014). Die Kookkurrenzdatenbank und Experimentierplattform CCDB (Belica 2007; Keibel/Belica 2007) umfasst nicht nur eine Sammlung von ca. 220.000 gespeicherten Kookkurrenzprofilen, sondern auch weitere Methoden, um diese auf ihre Ähnlichkeiten untereinander zu vergleichen. Auf die Ähnlichkeitsbewertung wiederum bauen weitere Methoden auf, die dazu konzipiert sind, Bedeutungsbereiche einzelner Wörter hervorzuheben (SOM-Methode, siehe auch Teichmann in diesem 
Heft) oder speziell die kontrastierenden Bereiche dicht beianderliegender Wörter (CNSMethode) bzw. die gemeinsamen Bereiche nicht ganz so dicht beieinanderliegender Wörter (CGC-Methode) herauszustellen (Vachková/Belica 2009). Während die Kookkurrenzanalyse mit COSMAS II viele Freiheiten eröffnet, gilt dies für die Arbeit mit den CCDB-Methoden nicht. In COSMAS II können aktuelle Daten oder eine Teilmenge als Datengrundlage gewählt werden, die Analysen der Kookkurrenzdatenbank basieren auf einem Datenbestand eines virtuellen Korpus mit Daten bis zum Jahr 2007. Das sprachliche Objekt, das zum Gegenstand der Untersuchung gemacht werden soll, kann in COSMAS II durch einen entsprechenden Suchausdruck vorgegeben werden, in der CCDB wird dies (in den allermeisten Fällen) als ein Lemma gedeutet, im Sinne einer Menge aller kleingeschriebenen flektierten Formen eines Paradigmas (z. B. effizient, effiziente, effizientes, effizienter, usw.). In der CCDB ist des Weiteren die analysierte Umgebung auf fünf Wörter vor und nach dem gefundenen Ausdruck innerhalb eines Satzes festgelegt, Umgebungswörter werden als Formen (nicht lemmatisiert) berücksichtigt. $\mathrm{Zu}$ diesen Vorgaben kann zwischen zwei Analyseergebnissen gewählt werden, für die Funktionswörter entweder ignoriert oder berücksichtigt wurden. Für letztere Ergebnisse stehen die Methoden, die die Ähnlichkeiten auswerten, allerdings nur über eine Expertenschnittstelle (expert UI) zur Verfügung.

\section{Freiheitsgrade bei der Analyse des Kontextverhaltens}

In COSMAS II lassen sich der zu analysierende Kontext und die Handhabung der Umgebungswörter stets neu individuell festlegen. Alle diese Optionen sind sinnvoll und ermöglichen die Formulierung unterschiedlicher Fragen an die jeweiligen Daten. Je nach Konstellation und Entscheidungen kann es aber sein, dass Analysen mit unterschiedlichen Vorgaben nicht mehr vorbehaltlos vergleichbar sind. Dies gilt für Analysen innerhalb von COSMAS II-Szenarien, aber auch gerade für diese gegenüber CCDB-Befunden.

Ohne an dieser Stelle zu sehr zu theoretisieren, sollen die naheliegenden Anwendungen von Profilvergleichen pragmatisch vereinfacht erörtert werden. Bei der Analyse des Kookkurrenzverhaltens kann das Ergebnis beeinflussen (a) die Wahl der zugrundegelegten Daten, (b) die Formulierung der Suchanfrage, (c) die Definition des zu analysierenden Kontextes und (d) weitere Parameter der Kookkurrenzanalyse. Prototypische Szenarien wären etwa, ein sprachliches Objekt in zwei verschiedenen Datenbeständen miteinander zu vergleichen oder die Ergebnisse zweier Objekte im selben Datenbestand gegenüberzustellen. Das linguistische Erkenntnisinteresse, wie Wörter in unterschiedlichen Texttypen (Quellen, Themen, Genre) typischerweise verwendet werden oder ob sich die typischen Verwendungsweisen im Laufe der Zeit verändern (als Indikator für Neubedeutungen), lässt sich so mit entsprechenden Korpora ${ }^{1}$ methodisch angehen. Vor diesem Hintergrund ergibt sich aber auch zwangsläufig, dass Kookkurrenzanalysen unter COSMAS II nur unter Vorbehalt mit denen der CCDB verglichen werden können, da der Datenbestand, auf dem letztere Analyse aufgesetzt hat, unter COSMAS II nicht zur Verfügung steht. Hinzu kommt, dass sehr große Treffermengen üblicherweise - wie in der $\mathrm{CCDB}^{2}$ auch - auf eine Zufalls-

Für letzteres wäre eine zeitlich gegliederte Reihe von ausreichend großen, gleichzeitig möglichst homogen zusammengesetzten Subkorpora erforderlich (vgl. Abb. 8).

2 Zusätzlich waren Kookkurrenzprofile zur Zeit der Erstellung der Kookkurrenzdatenbank aus technischen Gründen auf maximal 253 primäre Partnerwörter begrenzt, aktuelle Analysen können über 4.000 Einträge umfassen. 
stichprobe einer bestimmten Größe reduziert werden, was für die betroffenen Einträge im Nachhinein nicht mehr rekonstruiert werden kann.

\section{$2.1 \quad$ Aspekt ZeIT}

Das virtuelle Korpus der CCDB umfasst nur Daten bis zum Jahr 2007, das Gesamtarchiv inzwischen bis zum Jahr 2017, das Archiv des Paronymprojekts bis zum Jahr 2015. An dem Zeitverlauf ausgewählter Kandidaten für denkbare Wortkombinationen zeigt sich, wie stark die unterschiedlichen Zeitabschnitte der Daten auf das Ergebnis abfärben können.

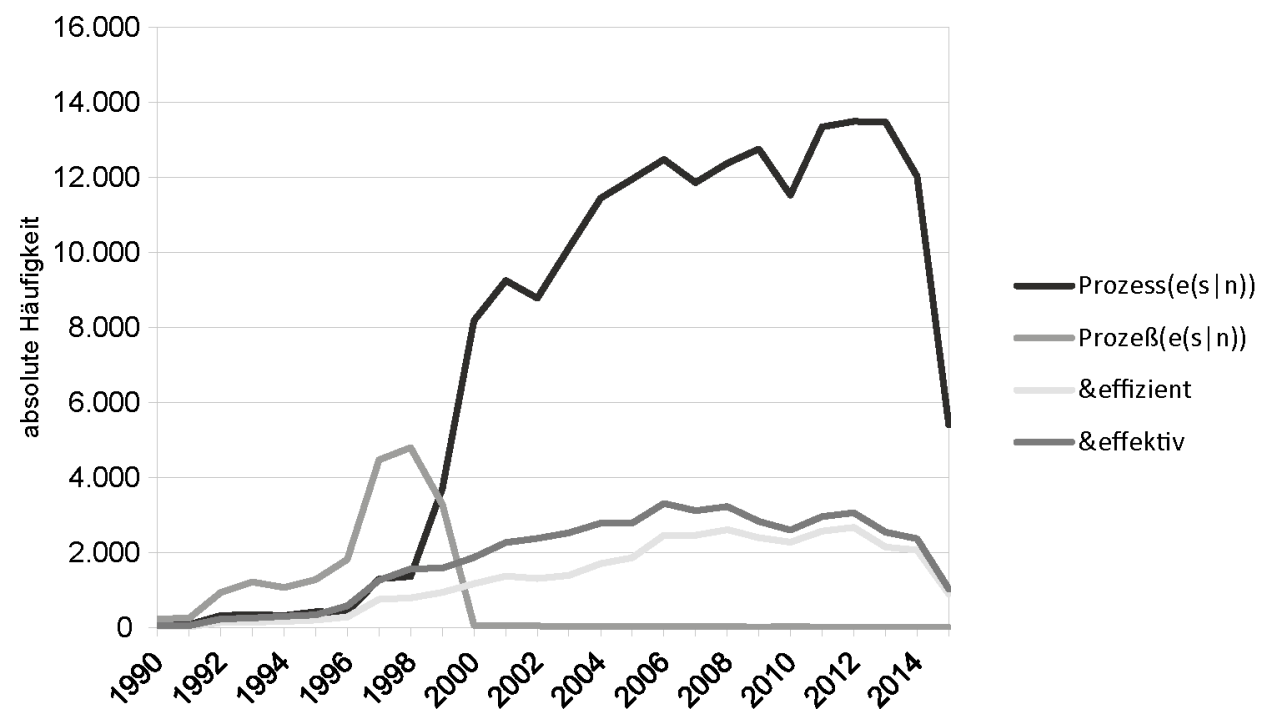

Abb. 1: Zeitverlauf ausgewählter Wörter im Paronymkorpus

Das in Abbildung 1 gezeigte Beispiel Prozess/ $\beta$ (inkl. zweifelhafter ß-Pluralformen) lässt sich aufgrund der Änderung der Schreibweise durch die Rechtschreibreform nachvollziehen und bei einer Analyse aus heutiger Sicht erahnen. In anderen Fällen können Veränderungen auch weniger dramatisch ausfallen, aber trotzdem Einfluss auf verschiedene Analysen haben, allein durch kurzzeitige Ausschläge bei Diskurshöhepunkten wie etwa Diskussionen zum Umgang mit Energie und Ressourcen. Der Vergleich des Verlaufs der Wörter des Paronympaars effizient/effektiv zeigt ein harmonisches Auf und Ab, sodass zumindest dem ersten Anschein nach keine Hinweise auf verzerrende Diskursausschläge vorliegen.

\subsection{Aspekt Formen (I)}

Wäre der Suchausdruck eine konkrete Wortform unter Beachtung von Groß-/Kleinschreibung, so hätte die Kookkurrenzanalyse einen eindeutigen und einheitlichen Bezugspunkt. Aus verschiedenen Gründen hat sich aber als besser zu handhabender Ansatz etabliert, als Ausgangspunkt für die Analyse die Menge der flektierten Formen (inkl. der nicht-markierten Form) anzusetzen, ein sog. Lemma. Für die CCDB werden z. B. für Adjektive aus dieser Menge üblicherweise nur die kleingeschriebenen Varianten betrachtet. Der Lemmasuchausdruck in COSMAS II (z. B. „\&effizient“) betrachtet aber zunächst grundsätzlich auch großgeschriebene Varianten ${ }^{3}$ mit, die allerdings über händisches Eingreifen in

\footnotetext{
3 Unabhängig von den übrigen Einstellungen zur Suche.
} 
die zur Kontrolle angebotene Wortformenliste herausgefiltert werden können. Der Einfluss der großgeschriebenen Varianten kann von unterschiedlicher Qualität sein und sich unterschiedlich stark auswirken - von quasi nicht messbar bis hin zu völliger Verzerrung. Einen ersten Hinweis darauf liefert alleine schon der quantitative Anteil, der in nachstehender Abbildung für 175 ausgewählte Paronymkandidaten dargestellt ist.

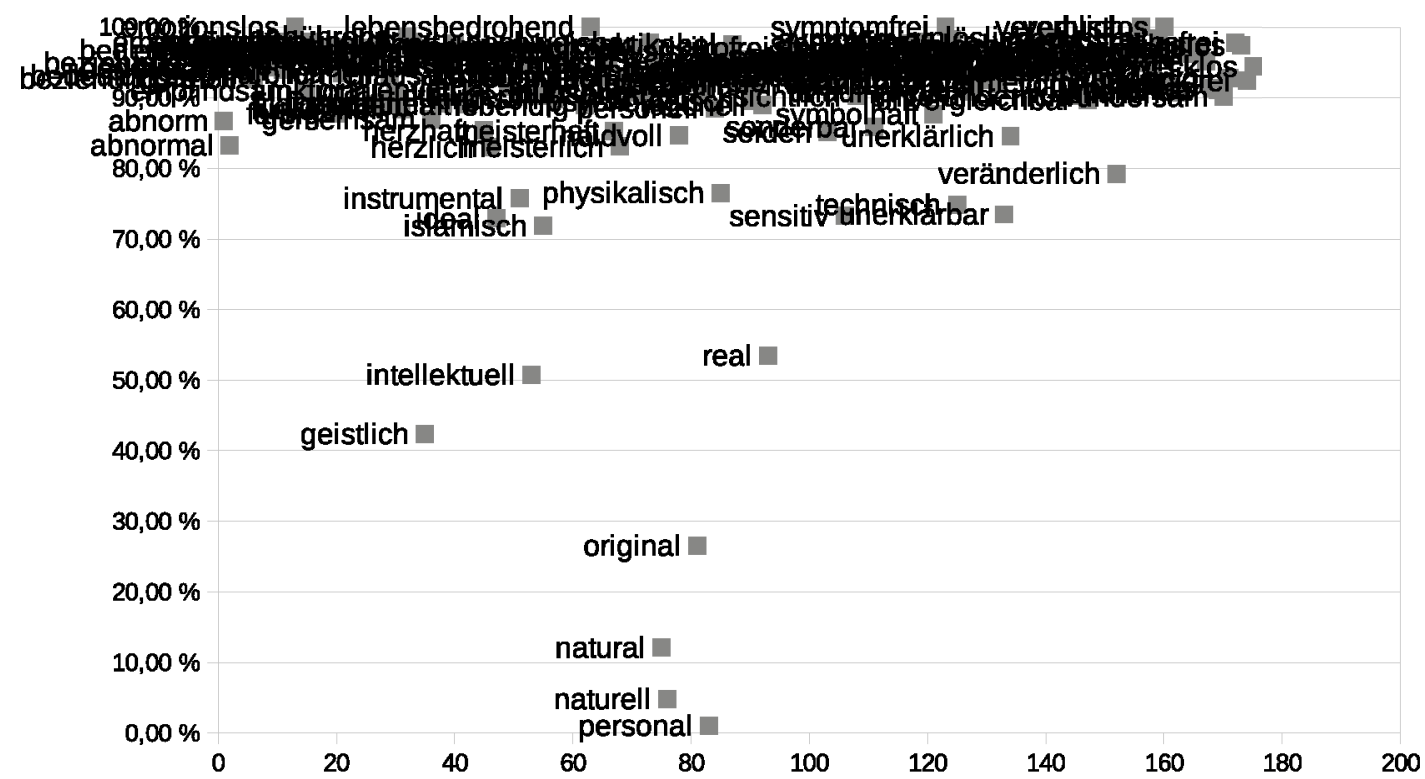

Abb. 2: Verteilung kleingeschriebene Formen vs. vom Lemmatisierer zugeordnete Formen

In Abbildung 2 ist aufgetragen, wie hoch der Anteil der kleingeschriebenen Formen an der Gesamtmenge der ungefiltert vom Lemmatisierer zugeordneten Formen im Paronymkorpus $^{4}$ ist. Die Darstellungsform als Streudiagramm eignet sich an dieser Stelle für unsere Zwecke besonders gut, da nicht der Großteil der Wörter interessiert, bei denen der Anteil einheitlich hoch (über 90\%) liegt. Es sind vielmehr die Exoten, auf die die Aufmerksamkeit gelenkt werden soll, bei denen es sich anders verhält: original, personal, geistlich, intellektuell, natural, naturell. Allein aufgrund der Zugehörigkeit zu einer bestimmten Wortklasse weisen Substantive naturgemäß andere Muster auf. Bei den ersten beiden Beispielen sieht man auf den ersten Blick, dass es sich um Einflüsse der Substantive handelt, bei den nächsten beiden Beispielen verbergen sich dahinter großgeschriebene Formen, die flektierten Formen des Adjektivparadigmas entsprechen: der Geistliche, die Intellektuellen. Gerade mit Blick auf die Gegenwörter (und im Vergleich zur CCDB) müsste bei Einbeziehung der großgeschriebenen Formen jeweils geprüft werden, wie stark der Einfluss - quantitativ und qualitativ - jeweils auf das Kookkurrenzverhalten ist. Bei den beiden letzten Beispielen verhält es sich etwas anders: Bei ihnen handelt es sich größtenteils um Teile fremdsprachiger Bezeichnungen von Institutionen, z. B. Museen, oder Musiktiteln, bei ersterem dominiert vom Englischen, bei letzterem vom Französischen.

\subsection{Aspekt „Thematische“ Zusammensetzung des Korpus}

Temporäre Diskursausschläge, aber auch kurzfristige Modeerscheinungen, können ein Analyseergebnis einseitig verzerren. In verallgemeinerter Form gilt dies grundsätzlich für die Zusammensetzung der Datengrundlage. Wenn eine Wortverbindung besonders cha-

4 Wie bei allen folgenden Recherchen/Analysen, sofern nicht explizit als CCDB-Resultate gekennzeichnet. 
rakteristisch für einen bestimmten Texttyp ist, dieser aber anteilsmäßig schwach zu der Datenmenge beiträgt, lässt sie sich nur schwerlich aufspüren - insbesondere wenn das Partnerwort ebenfalls für einen anderen Texttyp besonders charakteristisch ist, der deutlich stärker vertreten ist, in dem die Verbindung aber nur okkasionell belegt ist.

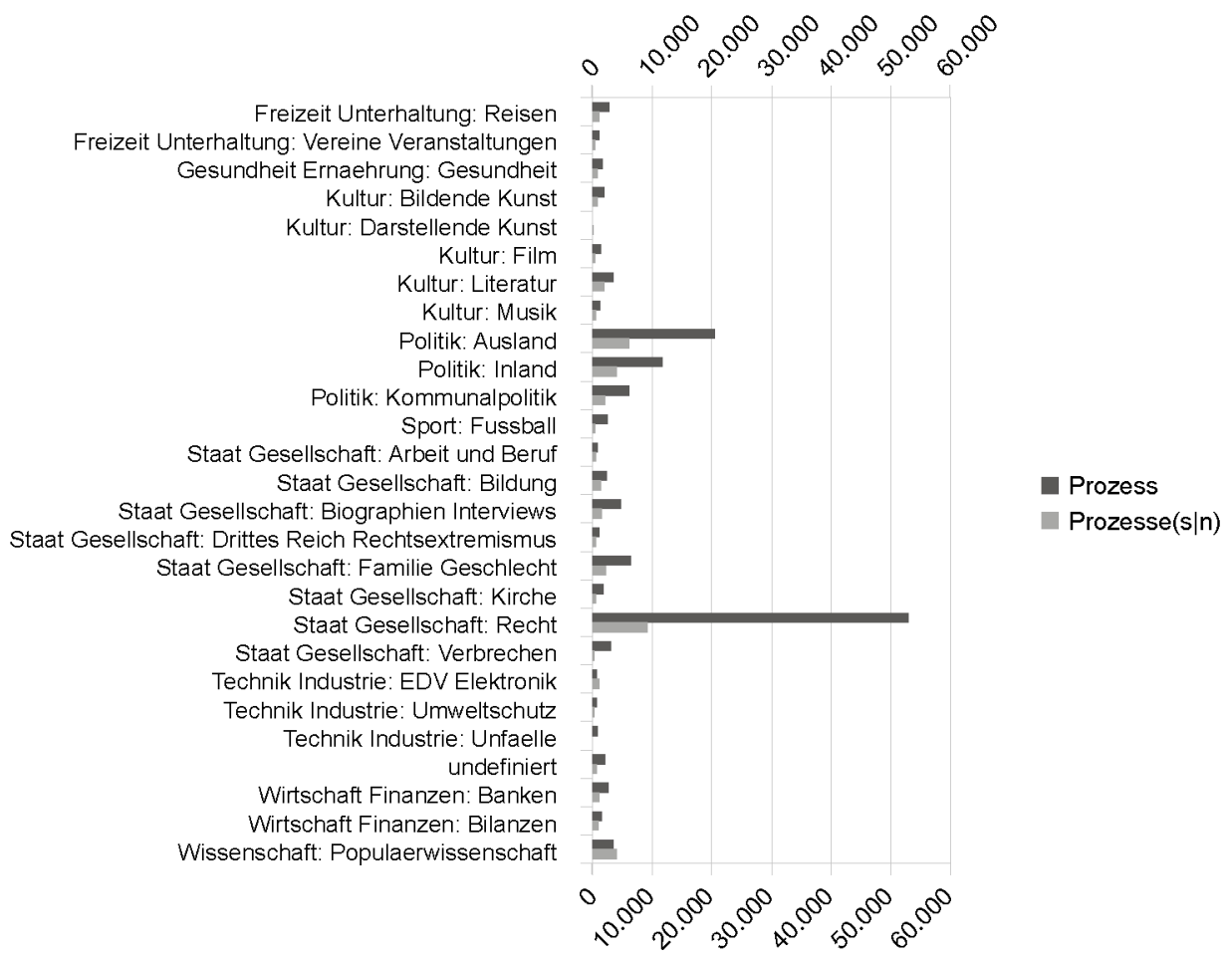

Abb. 3: Thematische Verteilung des Vorkommens von Prozess (Singular/Plural)

Dass das Wort effizient gerade im Umfeld von technischen Prozessen eine gewisse Rolle spielt, kann durchaus untergehen, da in absoluten Vorkommen die juristische Lesart von Prozess wesentlich mehr ins Gewicht fällt, dort aber nur in Einzelfällen mit effizient gemeinsam verwendet wird. Für die Zusammenstellung des CCDB-Korpus war gerade dies ein Beweggrund, Texttypen, die verhältnismäßig überrepräsentiert sind, bei der Stichprobenziehung ein wenig zu dämpfen.

\subsection{Aspekt Formen (II)}

Auch wenn in Abbildung 3 absolute Zahlen aufgetragen sind, so bleibt die Schieflage von Recht vs. Technik auch bei relativen Angaben bestehen. Ohne dies formal zu überprüfen, ist mit ziemlicher Sicherheit anzunehmen, dass das Wort Prozess eine hohe Charakterizität für das Thema Recht hat. Mit einer entsprechenden statistischen Bewertung ließe sich bestimmen, wie stark der beobachtete Wert vom erwartbaren abweicht, sodass Prozess als Schlüsselwort eingestuft werden kann. Nicht ohne Grund sind in Abbildung 3 die Werte für die Singular- und die Pluralformen getrennt dargestellt. Zwar wäre sicher auch das Lemma Prozess mit allen flektierten Formen als eine sprachliche Einheit ein Schlüsselwort, besonders stark zum Ausdruck kommt der Effekt aber vor allem bei den Singularformen. In diesem thematischen Umfeld ist es viel üblicher, über einen einzelnen Prozess zu berichten, als pauschale Aussagen zu formulieren, dass Prozesse als solche Eigenschaften hätten oder sich ändern müssten (z. B. effizienter werden müssten). 
Die Ermittlung typischer Wortverbindungen geht vollkommen analog vor, nur, dass nicht bewertet wird, wie oft ein Wort in Texten eines bestimmten Themas vorkommt, sondern wie oft es in der (meist sehr eng gefassten) lexikalischen Umgebung eines vorgegebenen Bezugswortes vorkommt. Um einen Eindruck davon zu bekommen, wie der obige Effekt auch hierbei wirkt, zeigt Abbildung 4, wie oft Formen des Adjektivs effizient und Formen des Substantivs Prozess in einem gemeinsamen Kontext von fünf Wörtern im Paronymkorpus vorkommen.

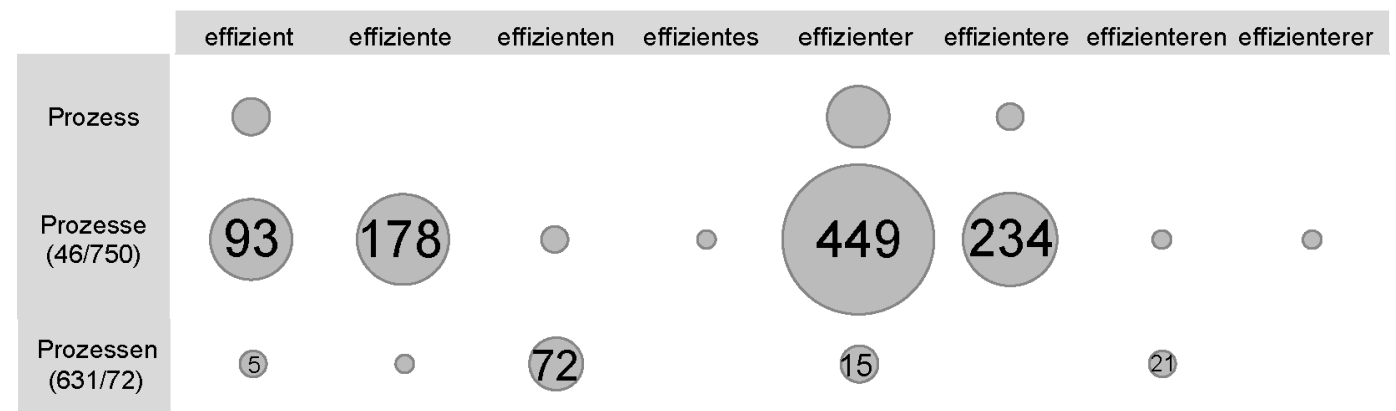

Abb. 4: Ko-Vorkommen der Formen von effizient und Prozess ${ }^{5}$

Den Schwerpunkt in dem Geflecht möglicher Beziehungen bilden rein quantitativ erkennbar an der Größe der Kreisfläche - verschiedene flektierte Formen des Adjektivs mit einer konkreten Pluralform des Substantivs, was sich auch bestätigt anhand der Bewertung formen-dedizierter Kookkurrenzanalysen, die bei Signifikanz als Beschriftung der Kreise angegeben ist. Ginge es bei der Untersuchung um das Substantiv - etwa im synonymischen/paronymischen Vergleich zu Prozedur oder Verfahren - sähe deren Verteilung aus möglicherweise zwei verschiedenen Gründen anders aus. Neben unterschiedlicher Singular-/Plural-Ausprägungen, die bei dem Wort Verfahren z. B. gar nicht oberflächlich erkennbar wären, schlägt sich natürlich der unterschiedliche Genus auf die Ausprägung der Flexionsendungen nieder (ein effizienter Prozeß, eine effiziente Prozedur, ein effizientes Verfahren), die allerdings wiederum so unterschiedlich synkretisch in den Paradigmen verteilt sind, dass kaum abzuschätzen ist, warum sich ihre Distribution wie verteilt und wie dies entsprechend zu interpretieren ist. Ein zweiter Punkt könnte sein, dass die Ausprägungen der verschiedenen Steigerungsstufen unterschiedlich stark vertreten sind. Dies kann besonders markant, etwa bei unmarkiertem Superlativ, durchscheinen (vgl. höchst, herzlichst, siehe Abb. 6), es kann aber auch Hinweise auf verstärkten Gebrauch von Komparativformen geben. Für die Beschreibung der Verwandtschaftsbeziehung zweier Wörter lassen sich die Unterschiede aufgrund der Flexionsendungen mit einem Verweis auf den unterschiedlichen Genus erklären. Unterschiede bei den Steigerungsstufen können aber ein Hinweis darauf sein, dass entweder eher absolute Zustände oder aber Veränderungen von graduellen Zuständen oder Vergleiche ausgedrückt werden.

Inwieweit der Befund relevant ist, lexikografisch hinterfragt, aufbereitet und beschrieben werden sollte, müsste jeweils im Einzelfall abgewogen werden. Verfolgen wir aber den Verdacht des besonderen Stellenwerts des Komparativs bei effizient exemplarisch noch

5 Kreisfläche entspricht Häufigkeit, angegebene Werte im Kreis: LLR von Adjektiv-Form in Umgebung von Substantiv-Form, Angaben in linker Spalte: Rang/LLR von Substantiv-Form in Umgebung von Adjektiv-Lemma. 


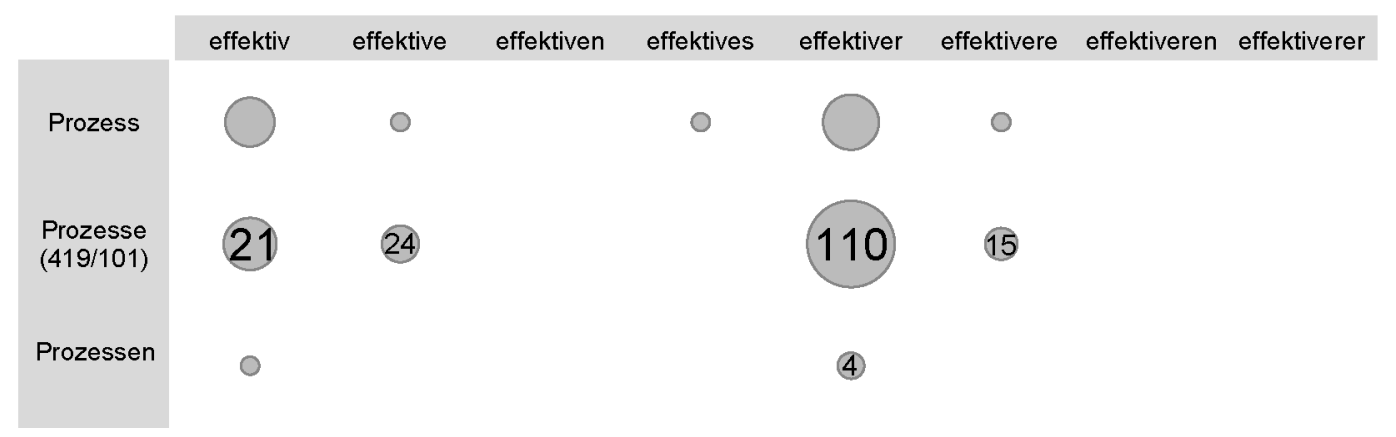

Abb. 5: Ko-Vorkommen der Formen von effektiv und Prozess

ein wenig weiter, zumal das Wort als Paronymgegenwort zu effektiv zu analysieren ist. Die Verteilung der Formbeziehungen zu effektiv und Prozess in Abbildung 5 zeigt - bis auf zwei Ausnahmen im Singular - eine Untermenge der Ausprägungen im Vergleich zu effizient. Sowohl die Häufigkeiten als auch die Auffälligkeiten spielen sich auf einem deutlich niedrigeren Niveau ab. Schwerpunkt ist allerdings auch hier die Form, die die Endung -er aufweist, bei der jedoch nicht aufzulösen ist, ob es sich um eine Flexionsendung oder die unmarkierte Form des Komparativs handelt. Da jedoch die flektierten Komparativendungen im Vergleich zu effizient erkennbar dünner besetzt sind, liegt die Vermutung nahe, dass sich auch nur ein geringer Anteil an Komparativformen in der Menge der synkretischen Formen verbirgt.

Einen weiteren Eindruck vermittelt die Darstellung in Abbildung 6, die zusammengefasst die Verteilung aller Formen für die oben bereits genannten 175 Paronymkandidaten visualisiert.

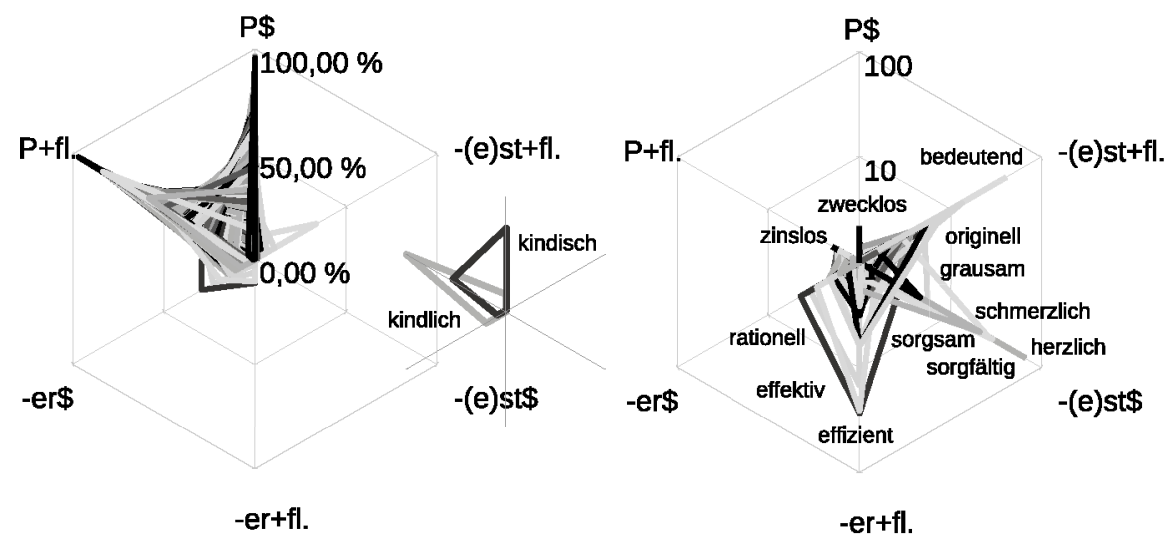

Abb. 6: Verteilung flektierter Formen prozentual pro Wort bzw. im Verhältnis zum Durchschnitt ${ }^{6}$

Dazu wurden die Formen unterschieden und gruppiert als die jeweils nicht weiter markierte Positiv-, die unklare „-er" - und die Superlativform, dazwischen jeweils die Entsprechungen mit Flexionsendungen aggregiert, um Genusunterschiede auszublenden. Im lin-

6 Von oben gegen den Uhrzeigersinn: Positiv unmarkiert, positiv flektiert außer -er, -er, -er plus Flexion, -(e)st, -(e)st plus Flexion; auf den sechs Strahlen sind die jeweiligen Werte für die Gruppen aufgetragen, die zu einer Form verbunden sind. Der äußere Rahmen des linken Diagramms der anteiligen Verhältnisse ist naturgemäß auf $100 \%$ begrenzt, die Formen zeigen hier die ungleichmäßige Verteilung. Im rechten Diagramm der Abweichungen vom Durchschnitt würde sich ein prototypisches Verhalten (= minimale Abweichungen vom Durchschnitt) als harmonisches Sechseck zeigen. Da hier die Skalierung aber durch die extremen Ausreißer bedingt ist, wären diese nur erkennbar, wenn wir in den zentralen Bereich um den Mittelpunkt hineinzoomen. 
ken Diagramm ist aufgetragen, wie hoch der prozentuale Anteil der Kategorie an der Gesamtmenge aller Formen eines Wortes ist. Es zeigt sich, dass bei den meisten Kandidaten der Bereich der Positivformen (unmarkiert und flektiert) am stärksten ausgeprägt ist, wohlweislich aber mit sehr vielen Schattierungen von einem 100\%-Extrem zum anderen. Für bestimmte Kandidatenpaare wäre es durchaus auch interessant, deren Muster miteinander zu vergleichen (siehe kindlich vs. kindisch). Um hervorzuheben, welche Kandidaten sich besonders auffällig verhalten, stellt das rechte Diagramm dar, wie sehr der beobachtete Anteil vom durchschnittlichen Anteil abweicht. Es bestätigt sich der Verdacht, dass sich das Wort effizient besonders ungewöhnlich verhält, sein Gegenwort effektiv steht ihm allerdings auch nicht wenig nach.

\subsection{Aspekt BetrachtungSRICHTUNG}

Theoretisch ließe sich der Einfluss der besonderen Verteilung untersuchen, indem entweder ganz viele Wörter (als Formen oder als Lemmata) auf typische Partnerwortformen analysiert werden und dann querschnittsartig betrachtet wird, welche Partnerwortform in welchen Analysen vorkommt. In der Kookkurrenzdatenbank gäbe es diese Möglichkeit durch die reziproke Suche, die sich allerdings nicht mit den dort angebotenen weiteren Vergleichsmöglichkeiten verbinden lässt. Abgesehen davon sind die diskutierten Wortverbindungen in dem Datenbestand (aufgrund zeitlicher und thematischer Zusammensetzung) (noch) nicht auffällig. Im Paronymkorpus lassen sie sich jedoch nachweisen, dafür ist in dem Szenario unklar, welche Reihen von Analysen zugrunde gelegt (und auch pragmatisch: wie verwaltet) werden sollen.

Die sprachlichen Einheiten, die betrachtet und auch miteinander verglichen werden sollen, stehen konzeptuell für die Gesamtheit aller Formen ihrer Paradigmen. Ein Hinweis auf eine atypische quantitative Zusammensetzung des Formenspektrums, wie bei den Beispielen angedeutet, wäre eine aufschlussreiche Information bei der Beschreibung, aber auch für die Sensibilisierung bei der weiteren Auswertung. Getrennte Analysen für alle verschiedenen Formen wären nicht nur extrem aufwendig, sie würden das Problem auch nur halb lösen, da ihre Ergebnisse später doch wieder zu einem Gesamtbild zusammengefügt werden müssten. Für den Abgleich mit Erkenntnissen anhand der CCDB-Methoden (sofern sonst plausibel) wäre es eine weitere einschränkende Facette. Als pragmatische Lösung ist es vollkommen legitim, das Kookkurrenzverhalten ausgehend von einem Lemma zu untersuchen. So wie Wörter bestimmter Wortklassen unterschiedlich auf die Einstellungen der Kookkurrenzanalyse (Kontext, Handhabung der Kontextwörter) reagieren, so unterschiedliche Spuren hinterlassen auch die Vertreter etwa der oben eingeführten Gruppen aus einem Adjektivflexionsparadigma. Flektierte, also attributiv verwendete Adjektivformen finden sich auf wenigen Positionen vor bindenden Substantiven, prädikativ, unmarkierte Formen stehen mit deutlicherem Abstand nach diesen, eventuell sogar dichter an ebenso typischen Verbformen oder Funktionswörtern. Insbesondere letzter Punkt gilt analog für Steigerungskonstruktionen. Zugegebenermaßen lassen sich Analysen mit Funktionswörtern häufig weniger intuitiv interpretieren. Um einen besseren Blick auf die positionellen und konstruktionellen Ausprägungen zu bekommen, bietet sich an, die Autofokus-Angaben und die syntagmatischen Muster näher zu betrachten. Ohne Autofokus würden die gleichen quantitativen Verhältnisse z. B. bei (engem) attributivem oder (weitem) prädikativem Gebrauch gleichermaßen bewertet. Die Option „Autofokus“ belohnt die engere Bindung mit einer höheren Bewertung und liefert gleichzeitig die Angabe der positionellen Präferenzen (Spalten „li/re“ in Abb. 7). Das syntagmatische 
Muster rekonstruiert die dominierende konkrete Ausprägung der Konstruktion. Alle drei Informationen liefern Hinweise, inwieweit das Zusammenspiel bestimmter Formen beim Vergleich des Kookkurrenzverhaltens eine besondere Berücksichtigung finden sollte. Für die Vergleichsmethoden innerhalb der CCDB fließt davon allerdings nur die AutofokusGewichtung der Beziehung als kleines Korrektiv der Ränge mit ein. Um den Zusammenhang genauer zu ergründen, empfiehlt sich ein Blick auf die syntagmatischen Muster. Selbst beim schnellen, oberflächlichen Betrachten fällt die breite Präsenz bestimmter Formen ins Auge. Bei der Kookkurrenzanalyse zu dem Lemma effizient zeigt sich die Form effizienter u. a. im syntagmatischen Muster des Partnerworts Prozesse.

Suchausdruck: \&effizient

\begin{tabular}{|l|c|c|l|l|r|l|l|}
\hline & Rk & LLR & $\#$ & li & re & Partnerwort & syntagmatisches Muster \\
\hline$Ð$ & 46 & 750 & 137 & -5 & 4 & Prozesse & $42 \%$ die Prozesse [... und] effizienter zu|und|gestalten \\
\hline
\end{tabular}

Suchausdruck: \&effektiv

\begin{tabular}{|l|l|l|l|l|l|l|l}
\hline & 421 & 101 & 35 & -4 & 1 & Prozesse & $54 \%$ die Prozesse $[\ldots]$ effektiver zu|gestalten|und
\end{tabular}

Suchausdruck: effizienter

\begin{tabular}{|l|r|r|r|r|l|l|l|l|}
\hline$\Psi$ & 36 & 449 & 65 & -5 & -1 & Prozesse & $89 \%$ die Prozesse [... und] effizienter zu|und|gestalten \\
\hline
\end{tabular}

Suchausdruck: effektiver

\begin{tabular}{|l|l|l|l|l|l|l|l|l|}
\hline & 105 & 110 & 20 & -4 & -1 & Prozesse & $95 \%$ die Prozesse [...] effektiver zu|gestalten|und \\
\hline
\end{tabular}

Abb. 7: Ausprägungen syntagmatischer Muster bei verschiedenen Kookkurrenzanaly sen

Die Mehrdeutigkeit der Endung -er lässt sich nicht mit einfachen Mitteln in den Griff auflösen. Das syntagmatische Muster legt aber doch stark die komparative Verwendung in der Konstruktion nahe. Der Anteil der Realisierungen beträgt allerdings nur $42 \%$, im Vergleich dazu liegt der Anteil beim Lemma effektiv sogar bei $54 \%$. Ob in den übrigen Textstellen die Konstruktion eher durch eine andere Wortstellung oder durch eine andere Form abweicht, lässt sich mit einer Kookkurrenzanalyse zu den jeweiligen konkreten Formen gegenprüfen. Auch dabei zeigt sich eine sehr starke Tendenz zur komparativen Konstruktion mit $89 \%$ bzw. $95 \%$.

Auch wenn sich die Häufigkeiten von effizient und effektiv deutlich unterscheiden und sich Muster deshalb auch unterschiedlich stark abzeichnen, ist doch festzuhalten, dass komparative Konstruktionen bei beiden gleichermaßen gut vertreten sind, was mit weiteren Partnerwörtern noch bestätigt werden kann. Ein Unterschied zwischen den beiden Wörtern, der gelegentlich postuliert wird, effektiv sei eine absolute Zuschreibung, während effizient eine graduelle Attribution sei, lässt sich nicht bestätigen. Während es bei letzterem um die (unterschiedlich) geschickte Nutzung von Ressourcen geht, wird ersteres mit einer (vorhandenen oder nicht vorhandenen) Wirkung verbunden. Nur ist es im Sprachgebrauch so, dass Wirkung nicht nur absolut betrachtet wird, sondern auch im Sinne von "stärkerer Wirkung" verglichen werden kann. Ob sich dies durch Paarformeln der Art effektiver und effizienter und auch womöglich erst im Laufe der Zeit verstärkt hat, müsste durch weitere Untersuchungen hinterfragt werden. Einen kleinen Eindruck möglicher Verschiebungen vermittelt Abbildung 8, in der der Anstieg oder auch Abfall der Ränge verschiedener ausgewählter Partnerwörter zu dem Lemma effizient visualisiert ist. Dies zeigt zum einen eine weitere interessante Anwendung des Vergleichs von Kookkurrenzprofilen. Es verdeutlicht zum anderen aber auch noch einmal, wie stark sich das Kookkur- 
renzverhalten in der Zeit, vermutlich abhängig von diskursiven Ausschlägen, möglicherweise aber auch von weiteren Einflüssen, verändern kann.

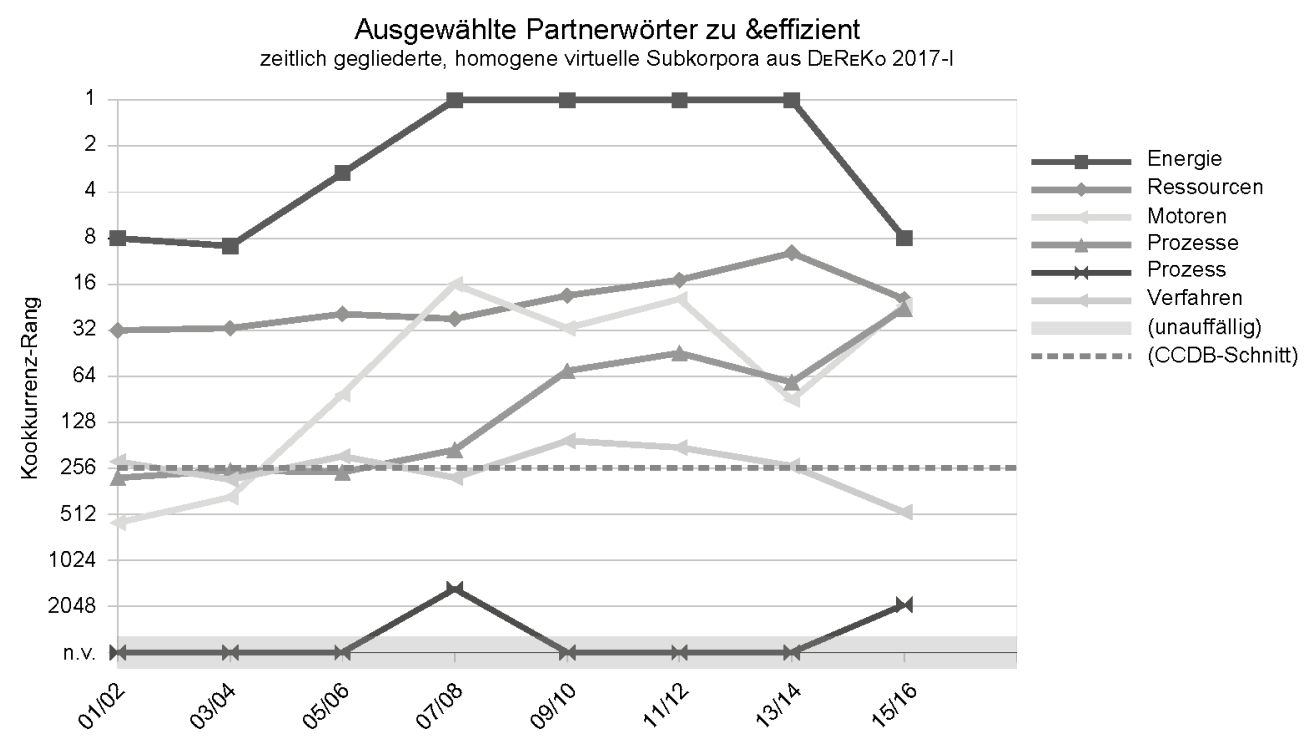

Abb. 8: Veränderungen des Kookkurrenzverhaltens im Laufe der Zeit

\section{3. $\quad$ Fazit}

Auswertungen und Vergleiche von Zeitverläufen, Themenverteilungen und Kookkurrenzverhalten liefern viele Hinweise auf Verwandtschaftsbeziehungen zwischen Wörtern; sie leisten wertvolle Beiträge, auch bei der Analyse von Paronymkandidaten. Selbst konfigurierbare Kookkurrenzanfragen an den aktuellen Datenbestand ermöglichen viele verschiedene Frageformulierungen auf Kosten fehlender weiterer Unterstützung bei der Auswertung. Eine derartige bietet zwar die Kookkurrenzdatenbank mit ihren Vergleichsmethoden, die allerdings durch ihren zugrunde gelegten Datenbestand und die voreingestellten Optionen keinen eigenen Spielraum mehr eröffnet, was wiederum den Vergleich mit eigenständigen Analysen erschwert. Aus pragmatischen Gründen bietet es sich an, mit Lemmata als Bezugsgrößen zu beginnen, wobei insbesondere bei diesen festzuhalten ist, dass ein Unterschied im Kookkurrenzverhalten im einfachen Sinne des Vorkommens/Nichtvorkommens oder des unterschiedlichen Rangs der Partnerwörter nicht zwingend bedeuten muss, dass dies auf einen semantischen Unterschied zurückzuführen ist. Es kann dafür u. a. auch einfach eine unterschiedliche Dominanz bestimmter Teilparadigmen verantwortlich sein, die sich teilweise bereits in der Gesamtformenverteilung abzeichnet. Dieser Umstand kann natürlich subtil zu der „Bedeutung“ beitragen. Für die genauere Untersuchung liegt es nahe, formenbezogene Analysen durchzuführen und die syntagmatischen Muster und die positionellen Präferenzen auszuwerten. Inwieweit diese Befunde jedoch lexikografisch relevant sind und sich leicht beschreiben lassen, sei dahingestellt. Auf auffällige Verwendungspräferenzen sollte aber allemal hingewiesen werden.

\section{Literatur}

Belica, Cyril (1995): Statistische Kollokationsanalyse und -clustering. Korpuslinguistische Analysemethode. http://corpora.ids-mannheim.de (Stand: 18. 12.2017).

Belica, Cyril (2007): Kookkurrenzdatenbank CCDB - V3. Eine korpuslinguistische Denk- und Experimentierplattform für die Erforschung und theoretische Begründung von systemisch-strukturellen Eigenschaften 
von Kohäsionsrelationen zwischen den Konstituenten des Sprachgebrauchs. http://corpora.ids-mannheim.de/ ccdb (Stand: 14. 6.2018)

Belica, Cyril (2011): Semantische Nähe als Ähnlichkeit von Kookkurrenzprofilen. In: Abel, Andrea/Zanin, Renata (Hg.): Korpora in Lehre und Forschung. Bozen-Bolzano: Freie Universität. S. 155-178.

Belica, Cyril/Perkuhn, Rainer (2015): Feste Wortgruppen/Phraseologie I: Kollokationen und syntagmatische Muster. In: Haß, Ulrike/Storjohann, Petra (Hg.): Handbuch „Wort und Wortschatz“. Berlin/Boston: De Gruyter. S. 201-225. (= Handbücher Sprachwissen 3).

Bodmer Mory, Franck (2014): Mit COSMAS II $»$ in den Weiten der IDS-Korpora unterwegs «. In: Institut für Deutsche Sprache (Hg.): Ansichten und Einsichten. 50 Jahre Institut für Deutsche Sprache. Redaktion: Melanie Steinle und Franz Josef Berens. Mannheim: Institut für Deutsche Sprache. S. 376-385.

Institut für Deutsche Sprache (2017a): Deutsches Referenzkorpus/Archiv der Korpora geschriebener Gegenwartssprache 2017-I (Release vom 8.3.2017). Mannheim: Institut für Deutsche Sprache. www.ids-mannheim. de/DeReKo (Stand: 14. 6.2018).

Institut für Deutsche Sprache (2017b): COSMAS II-Recherchesystem. https://cosmas2.ids-mannheim.de/cos mas2-web (Stand: 18. 12.2017)

Keibel, Holger/Belica, Cyril (2007): CCDB: A corpus-linguistic research and development workbench. In: Proceedings of Corpus Linguistics 2007. Birmingham University. http://corpus.bham.ac.uk/corplingprocee dings07/paper/134_Paper.pdf (Stand: 18.12.2017).

Kupietz, Marc et al. (2010): The German reference Corpus DeReKo: A primordial sample for linguistic research. In: Calzolari, Nicoletta et al. (Hg.): Proceedings of the Seventh International Conference on Language Resources and Evaluation (LREC 2010). Valletta, May 17-23, 2010. European Language Resources Association (ELRA). S. 1848-1854.

Kupietz, Marc/Schmidt, Thomas (2015): Schriftliche und mündliche Korpora am IDS als Grundlage für die empirische Forschung. In: Eichinger, Ludwig M. (Hg.): Sprachwissenschaft im Fokus. Positionsbestimmungen und Perspektiven. Berlin/Boston: De Gruyter. S. 297-322. (= Jahrbuch des Instituts für Deutsche Sprache 2014).

Perkuhn, Rainer (2007a): Systematic Exploration of Collocation Profiles. In: Proceedings of the $4^{\text {th }}$ Corpus Linguistics Conference (CL 2007), Birmingham. Birmingham: University of Birmingham.

Perkuhn, Rainer (2007b): „Corpus-driven“: Systematische Auswertung automatisch ermittelter sprachlicher Muster. In: Kämper, Heidrun/Eichinger, Ludwig M. (Hg.): Sprach-Perspektiven. Germanistische Linguistik und das Institut für Deutsche Sprache. Tübingen: Narr. S. 465-491. (= Studien zur Deutschen Sprache 40).

Perkuhn, Rainer/Keibel, Holger (2009): A brief tutorial on using collocations for uncovering and contrasting meaning potentials of lexical items. In: Minegishi, Makoto/Kawaguchi, Yuji (Hg.): Working papers in corpusbased linguistics and language education 3. Tokyo: Tokyo University of Foreign Studies. S. 77-91. (= TUFS).

Storjohann, Petra/Schnörch, Ulrich (2014): Empirical Approaches To Paronyms. In: Abel, Andrea/Vettori, Chiara/ Ralli, Natascia (Hg.): Proceedings of the XVI EURALEX International, Congress: The User in Focus. 15.19. July 2014, Bolzano/Bozen. Bozen: Institute for Specialised Communication and Multilingualism. S. 463-476.

Teubert, Wolfgang/Belica, Cyril (2014): Von der linguistischen Datenverarbeitung am IDS zur „Mannheimer Schule der Korpuslinguistik“" In: Institut für Deutsche Sprache (Hg.): Ansichten und Einsichten. 50 Jahre Institut für Deutsche Sprache. Redaktion: Melanie Steinle und Franz Josef Berens. Mannheim: Institut für Deutsche Sprache. S. 298-319.

Vachková, Marie/Belica, Cyril (2009): Self-organizing lexical feature maps. semiotic interpretation and possible application in lexicography. In: Interdisciplinary Journal for Germanic Linguistics and Semiotic Analysis 13, 2. S. 223-260

Rainer Perkuhn

Institut für Deutsche Sprache Mannheim

$\mathrm{R} 5,6-13$

68161 Mannheim

E-Mail: perkuhn@ids-mannheim.de 